\title{
SOLUCIÓN LOCAL PARA UNA ECUACIÓN HIPERBÓLICA NO LINEAL
}

\section{Oswaldo Ramos Chumpitaz}

RESUMEN.- Probamos la existencia de una solución local débil para el problema

$$
\mid \begin{aligned}
& u^{\prime}-M(u) \Delta u-u^{3}=f \\
& u(0)=u_{0} \\
& u^{\prime}(0)=u_{1}
\end{aligned}
$$

donde $M(u)=1+\sqrt{a(u)}$ siendo $a(u)=a(u, u)$ donde $a(u, v)=\int_{\Omega} \nabla u . \nabla v d x$ es la forma de Dirichlet, $\Omega \subset \mathbb{R}^{3}$ es abierto acotado de frontera bien regular.

\section{INTRODUCCIÓN}

En este trabajo probamos la existencia de una solución débil local para el problema asociado a la ecuación hiperbólica no lineal

$$
\frac{\partial^{2} u}{\partial t^{2}}-M(u) \Delta u-u^{3}=f
$$

donde

$$
M(u)=1+\sqrt{a(u)}
$$

siendo

$$
a(u)=a(u, u), a(u, v)=\int_{\Omega} \nabla u \cdot \nabla v d x
$$

la forma de Dirichlet, $\nabla$ y $\Delta$ son los operadores gradiente y laplaciano, respectivamente.

Si en la ecuación (1) se tuviera término no lineal $+u^{3}$, entonces se puede resolver aplicando el método de la energía por el comportamiento monótono del término no lineal, sin embargo debido al signo negativo del término cúbico este método no es aplicable, motivo por el cual hacemos uso del método «pozo de potencial» introducido por Sattinger [9], combinado con el método de Galerkin. La ecuación (1) describe las vibraciones transversales de una cuerda elástica. 
Problemas similares fueron estudiados por Pohozaev [8], Lions [4], Medeiros [5], Dickey [2], Ebihara [3] y Medeiros - Milla [ 6 ] y [ 7 ].

\section{PRELIMINARES}

Sea $\Omega \subset \mathbb{R}^{3}$ abierto limitado de frontera $\Gamma$ bien regular, $\|\cdot\|$ norma en $H^{1}(\Omega) ;|\cdot|_{q}$ norma en $L^{q}(\Omega)$.

LEMA 1 (Sobolev).- Si $1 \leq q \leq 6 \Rightarrow H_{0}^{1}(\Omega) \subset L^{q}(\Omega)$ es contínua. Definimos las energías cinética y potencial asociadas a nuestra ecuación por:

$$
\begin{aligned}
& K(\omega)=\frac{1}{2}|\omega|^{2}, \omega \in L^{2}(\Omega) \\
& J(z)=\frac{1}{2}\|z\|^{2}+\frac{1}{3}\|z\|^{3}-\frac{1}{4}|z|_{4}^{4}, z \in H_{0}^{1}(\Omega)
\end{aligned}
$$

Además, definimos el número $d$ por

$$
d=\inf _{\substack{u \in H_{0}^{1}(\Omega) \\ u \neq 0}}\left\{\max _{\lambda \geq 0} J(\lambda u)\right\}
$$

$d$ es la profundidad del «pozo de potencial», $\lambda_{1}(u)$ el primer valor para el cual $J(\lambda u)$ posee un máximo.

LEMA 2.- Sea d estrictamente positivo, esto es, $d>0$. Definimos ahora nuestro pozo potencial $W$ por:

$$
W=\left\{u \in H_{0}^{1}(\Omega) / J(\lambda u)<d, \forall 0 \leq \lambda \leq 1\right\}
$$

Observación.- si $u \in W$, se tiene que $J(\lambda u) \geq 0$ y $\lambda_{1}(u)>1$.

LEMA 3 (Sattinger).- $W$ es un conjunto abierto acotado de $H_{0}^{1}(\Omega)$.

TEOREMA 1.- Sea $\Omega$ abierto acotado bien regular de $\mathbb{R}^{3}, T>0$. Además,

i ) $f \in L^{2}\left(0, T ; H_{0}^{1}(\Omega)\right) \cap L^{1}\left(0, T ; L^{2}(\Omega)\right)$

ii ) $u_{0} \in H_{0}^{1}(\Omega) \cap H^{2}(\Omega) \cap W$

iii) $u_{1} \in H_{0}^{1}(\Omega)$

iv) $E_{0}+2 \sqrt{E_{0}+\left(\int_{0}^{T}|f(t)| d t\right)^{2}} \int_{0}^{T}|f(t)| d t<d ; E_{0}=K\left(u_{1}\right)+J\left(u_{0}\right)$

Entonces existen $T_{0}$ real tal que $0<T_{0}<T$ y una función $u$ que satisfacen: 


$$
\begin{aligned}
& u \in L^{\infty}\left(0, T_{0} ; H_{0}^{1}(\Omega) \cap H^{2}(\Omega)\right) \\
& u^{\prime} \in L^{\infty}\left(0, T_{0} ; H_{0}^{1}(\Omega)\right) \\
& u^{\prime \prime} \in L^{2}\left(0, T_{0} ; L^{2}(\Omega)\right)
\end{aligned}
$$

y $u$ es solución débil de

$$
\mid \begin{aligned}
& u^{\prime \prime}-(1+\sqrt{a(u)}) \Delta u-u^{3}=f \\
& u(0)=u_{0} \\
& u^{\prime}(0)=u_{1}
\end{aligned}
$$

\section{Demostración}

Etapa 1.- Soluciones aproximadas

Sea $\left\{w_{1}, w_{2}, \ldots\right\}$ base de $H_{0}^{1}(\Omega) \cap H^{2}(\Omega)$ formada por las funciones propias de $-\Delta$, esto es $-\Delta w_{j}=\lambda_{j} w_{j}$

$$
u_{m}(t)=\sum_{i=1}^{m} g_{i n}(t) w_{i} \quad, \quad V_{m}=\left[w_{1}, \ldots, w_{m}\right], m \geq 1
$$

subespacio finito generado por $\left\{w_{1}, \ldots, w_{m}\right\}$.

Las funciones $g_{i m}(t)$ son determinadas por el sistema de ecuaciones diferenciales ordinarias no lineales

$$
(S . A) \mid \begin{aligned}
& \left(u_{m}^{\prime \prime}(t), w_{j}\right)+\left(1+\sqrt{a\left(u_{m}(t)\right)}\right) a\left(u_{m i}(t), w_{j}\right)-\left(u_{m}^{3}(t), w_{j}\right)=\left(f(t), w_{j}\right), \\
& u_{m}(0)=u_{0 m} \rightarrow u_{0} \quad \text { en } H_{0}^{1}(\Omega) \cap H^{2}(\Omega) \\
& u_{m}^{\prime}(0)=u_{1 m} \rightarrow u_{1} \quad \text { en } H_{0}^{1}(\Omega)
\end{aligned}
$$

La existencia de las funciones $g_{i m}(t)$ en un intervalo $\left[0, T_{m}\right]$ está garantizada por el Teorema de Caratheodory.

Las estimativas a priori que haremos permitirán extender estas funciones a un intervalo $[0, T]$ independiente de $m$.

\section{Etapa 2.-}

Estimativa a priori (i):

Como $J$ y. $K$ son funcionales continuos, entonces $E_{0 m} \rightarrow E_{0}$. Luego 


$$
E_{0 m}+2 \sqrt{E_{0 m}+\left(\int_{0}^{T}|f(t)| d t\right)^{2}} \int_{0}^{T}|f(t)| d t<d
$$

para $m$ grande multiplicando la ecuación de (S.A) por $g_{j m}^{\prime}(t)$ y sumando de 1 a $m$ obtenemos

$$
\frac{d}{d t}\left\{K\left(u_{m}^{\prime}(t)\right)+J\left(u_{m}(t)\right)\right\}=\left(f(t), u_{m}^{\prime}(t)\right)
$$

luego

$$
K\left(u_{m}^{\prime}(t)\right)+J\left(u_{m}(t)\right)=K\left(u_{1 m}\right)+J\left(u_{0 m}\right)+\int_{0}^{t}\left(f(s), u_{m}^{\prime}(s)\right) d s
$$

Probaremos que $u_{m}(t) \in W, \forall t \in\left[0, t_{m}\right]$.

Por reducción al absurdo, supongamos que $\exists t \in\left[\dot{0}, t_{m}\right]$ tal que $u_{m}(t) \in W$.

Sea $t^{*}=\inf \left\{t / u_{m}(t) \notin W\right\}$, siendo $u_{m}(t)$ contínua tenemos que $u_{m}\left(t^{*}\right) \in \partial W$, esto es,

$$
J\left(u_{m}\left(t^{*}\right)\right)=d
$$

Por otro lado, sea $N=\sup _{t \in\left[0, t^{*}\right]}\left|u_{m}^{\prime}(t)\right|$, entonces (4) implica

$$
\frac{1}{2} N^{2} \leq E_{0 m}+N \int_{0}^{T}|f(t)| d t
$$

de donde sigue que

$$
N \leq 2 \sqrt{E_{0 m}+\left(\int_{0}^{T}|f(t)| d t\right)^{2}}
$$

Ahora por (6) y (3)

$$
\begin{aligned}
J\left(u_{m}\left(t^{*}\right)\right) & \leq K\left(u_{m}^{\prime}\left(t^{*}\right)\right)+J\left(u_{m}\left(t^{*}\right)\right)=E_{0 m}+\int_{0}^{t^{*}}\left(f(t), u_{m}^{\prime}(t)\right) d t \\
& \leq E_{0 m}+N \int_{0}^{t^{*}}|f(t)| d t<d
\end{aligned}
$$

Así $J\left(u_{m}\left(t^{*}\right)\right)<d$ contradiciendo (5) luego $u_{m}(t) \in W, \forall t \in\left[0, t_{m}\right]$, entonces 


$$
\left\|u_{m}(t)\right\| \leq C \text { independiente de } m, \forall t \in\left[0, t_{m}\right] .
$$

También

$$
\begin{aligned}
\frac{1}{2}\left|u_{m}^{\prime}(t)\right|^{2} & \leq K\left(u_{m}^{\prime}(t)\right)+J\left(u_{m}(t)\right) \leq E_{0 m}+\int_{0}^{t}|f(s)|\left|u_{m}^{\prime}(s)\right| d s \\
& \leq C+\frac{1}{2} \int_{0}^{t}\left|u_{m}^{\prime}(s)\right|^{2} d s,
\end{aligned}
$$

luego por el lema de Gronwall

$$
\left|u_{m}^{\prime}(t)\right| \leq C \text { independiente de } m, \forall t \in\left[0, t_{m}\right]
$$

así podemos extender la solución $u_{m}(t)$ al intervalo $[0, T] \mathrm{y}$

$$
\left\|u_{m}(t)\right\| \leq C,\left|u_{m}^{\prime}(t)\right| \leq C \quad \forall t \in[0, T]
$$

\section{Estimativa a priori (ii):}

Multiplicando (S.A.) por $g_{j m}^{\prime}(t) \lambda_{j}$, sumando de 1 a $m$, y usando el Lema 1 tenemos

$$
\begin{aligned}
\frac{1}{2} \frac{d}{d t}\left\|u_{m}^{\prime}(t)\right\|^{2}+\frac{1}{2}\left(1+\sqrt{a\left(u_{m}(t)\right)}\right) \frac{d}{d t}\left|\Delta u_{m}(t)\right|^{2} & =\int_{\Omega}\left(\sum_{i=1}^{3} \frac{\partial}{\partial x_{i}} u_{m}^{3}(t) \frac{\partial}{\partial x_{i}} u_{m}^{\prime}(t)\right) d x+ \\
& +a\left(f(t), u_{m}^{\prime}(t)\right) \\
& \leq\|f(t)\|^{2}+C\left\{\left\|u_{m}^{\prime}(t)\right\|^{2}+\left|\Delta u_{m}(t)\right|^{2}\right\}
\end{aligned}
$$

Sean $\mu(t)=1+\left\|u_{m}(t)\right\|, \quad \beta(t)=\left\|u_{m}^{\prime}(t)\right\|^{2}, \quad \gamma(t)=\left|\Delta u_{m}(t)\right|^{2}$.

Tenemos entonces la desigualdad

$$
\beta^{\prime}(t)+\mu(t) \gamma^{\prime}(t) \leq\|f(t)\|^{2}+C\{\beta(t)+\gamma(t)\}
$$

de donde obtenemos

$$
\frac{\beta^{\prime}(t)}{\mu(t)}+\gamma^{\prime}(t) \leq\|f(t)\|^{2}+C\left\{\frac{\beta(t)}{\mu(t)}+\gamma(t)\right\}
$$

Si $h(t)=\frac{1}{\mu(t)} \beta(t)+\gamma(t) \Rightarrow h^{\prime}(t)=\frac{1}{\mu(t)} \beta^{\prime}(t)+\gamma^{\prime}(t) \frac{\mu^{\prime}(t) \beta(t)}{\mu^{2}(t)}$ 
42 SOLUCIÓN LOCAL PARA UNA ECUACIÓN ...

Luego

$$
\begin{aligned}
h^{\prime}(t)+\frac{\mu^{\prime}(t)}{\mu^{2}(t)} \beta(t) & \leq\|f(t)\|^{2}+C h(t) \\
h^{\prime}(t) & \leq\|f(t)\|^{2}+C h(t)+\frac{\left|\mu^{\prime}(t)\right|}{\mu^{2}(t)} \beta(t)
\end{aligned}
$$

tenemos

$$
h^{\prime}(t) \leq\|f(t)\|^{2}+C h(t)+C_{1} h^{\frac{3}{2}}(t)
$$

Multiplicando por $e^{-t}, t \geq 0$, transponiendo y simplificando se tiene

$$
h(t) \leq K_{1}+K_{2} \int_{0}^{t} h^{\frac{3}{2}}(s) d s
$$

donde

$$
K_{1}=h(0)+e^{C T} \int_{0}^{T}\|f(s)\|^{2} d s, \quad K_{2}=C_{1} e^{C T}
$$

Sea

$$
\begin{aligned}
& \varphi(t)=K_{1}+K_{2} \int_{0}^{t} h^{\frac{3}{2}}(s) d s \\
& \varphi(t)=K_{2} h^{\frac{3}{2}}(t) d s \quad \text { y como } h(t) \leq \varphi(t)
\end{aligned}
$$

entonces

$$
\varphi^{\prime}(t)=K_{2} \varphi^{\frac{3}{2}}(t)
$$

Luego transponiendo e integrando, sigue

$$
\varphi^{-\frac{1}{2}}(t)=\varphi^{-\frac{1}{2}}(0)-\frac{1}{2} K_{2} t \geq 0 \quad \forall t \in\left[0, \frac{2}{K_{1}^{\frac{1}{2}} K_{2}}\right]
$$

Sea $T_{0}$ tal que

$$
0<T_{0}<\frac{2}{K_{1}^{\frac{1}{2}} K_{2}}
$$

entonces, $\forall t \in\left[0, T_{0}\right]$

$$
h(t) \leq \varphi(t) \leq \frac{1}{\left(K_{1}^{\frac{1}{2}}-\frac{1}{2} K_{2} T_{0}\right)^{2}}=K_{3}
$$


luego

$$
\frac{\beta(t)}{\mu(t)}+\gamma(t) \leq K_{3}, \text { asi } \gamma(t)=\left|\Delta u_{m}(t)\right|^{2} \leq K_{3}, \forall t \in\left[0, T_{0}\right]
$$

También

$$
\frac{\beta(t)}{\mu(t)} \leq K_{3} \Rightarrow \beta(t) \leq K_{3} \mu(t) \Rightarrow\left\|u_{m}^{\prime}(t)\right\|^{2} \leq K_{3}\left(1+\left\|u_{m}\right\|\right) \leq K_{3}(1+C)
$$

así

$$
\left\|u_{m}^{\prime}(t)\right\| \leq K_{4} \quad\left\|u_{m}(t)\right\|_{H^{2}(\Omega)} \leq K_{5}, \quad \forall t \in\left[0, T_{0}\right]
$$

\section{Etapa 3.- Pasaje al limite.}

De las estimativas (9) y (10) tenemos que

$$
\begin{aligned}
& u_{m} \rightarrow u \text { débil estrella en } L^{\infty}\left(0, T ; H^{\frac{1}{2}}(\Omega)\right) \\
& u_{m}^{\prime} \rightarrow u \text { débil estrella en } L^{\infty}\left(0, T ; L^{2}(\Omega)\right) \\
& u_{m} \rightarrow u \text { débil estrella en } L^{\infty}\left(0, T_{0} ; H^{2}(\Omega)\right) \\
& u_{m}^{\prime} \rightarrow u^{\prime} \text { débil estrella en } L^{\infty}\left(0, T_{0} ; H_{0}^{1}(\Omega)\right) .
\end{aligned}
$$

Como las inmersiones $H^{2}(\Omega) \hookrightarrow H^{1}(\Omega) \hookrightarrow L^{2}(\Omega)$ son compactas, por el Teorema de Lions - Aubin se sigue que

$$
\begin{aligned}
& u_{m} \rightarrow u \text { fuerte en } L^{\infty}\left(0, T_{0} ; H_{0}^{1}(\Omega)\right) \\
& u_{m}^{\prime} \rightarrow u^{\prime} \text { fuerte en } L^{\infty}\left(0, T_{0} ; L^{2}(\Omega)\right) .
\end{aligned}
$$

Luego podemos pasar al límite en la ecuación aproximada y obtener la solución del problema. Las condiciones iniciales se verifican en forma estándar. 


\section{BIBLIOGRAFÍA}

[1] Aubin J. P. Un Theorema de Compacité. C. R. Acad. Science Paris, 256, 5042 - 5044 (1963).

[2] Dickey R. W. The initial value problem for a non linear semi infinite string. Proceding of the Royal Society of Edinbourg 82 A, 19 - 26 (1978).

[3] Ebihara Y. On solutions of semilinear wave equations non linear analysis. Vol 6 $\mathrm{N}^{\circ} 5,467-486$ (1982).

[4] Lions J. L. Quelques méthodes de resolutions de problémes aux limites non lineaires. Dunod Paris (1969).

[5] Medeiros L. A. On a new class of non linear wave equations. J. of Math Anal and Appl. Vol 69, No 1, 252 - 262 (1979).

[6] Medeiros L. A. Milla M.- Local solutions for a nonlinear unilateral Problem (to appear).

[7] Medeiros L. A. Milla M. Remarks on a nonlinear model vibrations of string with damping. $30^{\circ} \mathrm{SBA}, \mathrm{LNCC}-\mathrm{R} . J .(1989)$.

[8] Pohozaev S. On a class of quasilinear hyperbolic equations. Math. Sbornik, Vol. 96 (1975).

[9] Sattinger D. H. On global solution of nonlinear hyperbolic equations, Arch. Rat. Mech. Anal. 30, 148 - 172 (1968). 